\title{
Stereotactic radiosurgery for patients with breast cancer brain oligometastases - molecular subtypes and clinical outcomes
}

\author{
Ivica Ratosa ${ }^{\circledR}$, Marija Skoblar Vidmar \\ Division of Radiation Oncology, Institute of Oncology Ljubljana, Ljubljana, Slovenia
}

\begin{abstract}
Background: We sought to determine the clinical outcomes of patients with breast cancer (BC) who had undergone stereotactic radiosurgery (SRS) for a limited number of brain metastases (BM) and to identify factors influencing overall survival (OS) and local control.

Materials and methods: The records of 45 patients who underwent SRS for 72 brain lesions were retrospectively evaluated. Statistics included the chi-squared test, Kaplan-Meier method, and the multivariate Cox model.

Results: The median number of treated BM was 2 (range 1-10). Median OS from BM diagnosis and post-SRS were 27.6 [95\% confidence interval (Cl): $14.8-40.5)$ and 18.5 months (95\% Cl: 11.1-25.8), respectively. One-year and two-year survival rates after BM diagnosis were $55 \%$ and $41 \%$, respectively. In a univariate analysis, the Luminal-B-human-epidermal-growth-receptorpositive (HER2+) subtype had the longest median OS at 39.1 months ( $95 \% \mathrm{Cl}: 34.1-44.1, \mathrm{p}=0.004)$. In an adjusted analysis, grade 2 [hazard ratio (HR): $0.1 ; 95 \% \mathrm{Cl}: 0.1-0.6, \mathrm{p}=0.005$ ), craniotomy (HR: $0.3 ; 95 \% \mathrm{Cl}: 0.1-0.7 ; \mathrm{p}=0.006$ ), and $\geq 2$ systemic therapies received ( $\mathrm{HR}: 0.3 ; 95 \% \mathrm{Cl}: 0.1-0.9, \mathrm{p}=0.028$ ) were associated with improved OS. One-year and two-year intracranial progression-free survival rates were $85 \%$ and $63 \%$, respectively. Four factors for a higher risk of any intracranial recurrence remained significant in the adjusted analysis, as follows: age < 50 years (HR: 4.2; 95\% Cl: 1.3-36.3; $p=0.014$ ), grade 3 (HR: 3.7 ; 95\% Cl: 1.1-13.2; $\mathrm{p}=0.038), \mathrm{HER} 2+(\mathrm{HR}: 6.9 ; 95 \% \mathrm{Cl}: 1.3-36.3 ; \mathrm{p}=0.023)$, and whether the brain was the first metastatic site (HR: $4.7 ; 95 \% \mathrm{Cl}: 1.6-14.5 ; \mathrm{p}=0.006)$.

Conclusion: Intrinsic BC characteristics are important determinants for both survival and intracranial control for patients undergoing SRS for oligometastatic brain disease.

Key words: breast cancer; brain metastases; molecular subtype; stereotactic radiosurgery; radiation therapy

Rep Pract Oncol Radiother 2021;26(1):1-11
\end{abstract}

\section{Introduction}

Patients with breast cancer (BC) frequently suffer from the development of distant metastasis, with bone, lung, liver, and brain metastasis (BM) being the most common [1]. The incidence proportion for the development of BM among a subset of metastatic BC at diagnosis is around $7-16 \%[2$,
3] and approximately $17 \%$ during follow-up [3]. When different tumor subtypes are considered after a diagnosis of $\mathrm{BM}$, the highest percentage was observed among patients with hormonal receptorpositive human epidermal growth receptor-negative (HR+/HER2-) primary BC, reaching 30-45\%, followed by triple-negative (TNBC), HR+/HER2+, and HER2-positive subtypes with an incidence of

Address for correspondence: Ivica Ratosa, MD, PhD, Institute of Oncology Ljubljana, Zaloska cesta 2, SI-1000 Ljubljana, Slovenia; tel.: +386 15879 522; fax: +386 15879 304; e-mail: iratosa@onko-i.si

This article is available in open access under Creative Common Attribution-Non-Commercial-No Derivatives 4.0 International (CC BY-NC-ND 4.0) license, allowing to download articles and share them with others as long as they credit the authors and the publisher, but without permission to change them in any way or use them commercially 
$17-25 \%, 20-24 \%$, and $15-21 \%$, respectively [3-6]. In autopsy studies, up to $35 \%$ of patients with BC are found to have BM, not all of which had been clinically relevant in the lifetime of the patients [7]. The combination of new systemic treatment options, which have resulted in improved survival for metastatic BC and implementation of sensitive diagnostic imaging in clinical practice, have contributed to a higher overall detection of brain metastasis [8].

Local management of BM involves surgery, either single fraction or hypofractionated radiosurgery (SRS), and/or whole-brain radiotherapy (WBRT). Two large randomized studies comparing SRS alone versus SRS and WBRT found higher intracranial failure rates with SRS alone without compromising overall survival (OS) $[9,10]$. Studies also demonstrated significantly worse learning and memory functions with the SRS and WBRT combination compared to SRS alone, which made the latter a first-choice treatment, particularly for limited brain metastatic disease and, increasingly, for patients with four or more BM [11, 12]. Patients with diffuse brain involvement or large metastases are typically offered WBRT without or with hippocampal sparing to preserve the neurocognitive function $[11,13]$.

Roughly, $50 \%$ of patients with BC with BM will die from intracranial progression [3]. HER2-positive disease is more frequently associated with intracranial recurrence after brain-directed radiotherapy for BM, compared to HR+/HER2-primary $\mathrm{BC}$ molecular subtypes [6]. In contrast, patients with TNBC disease demonstrated higher rates of new brain lesions and shorter time to salvage SRS or WBRT [6].

The purpose of this retrospective study was to assess the clinical outcomes of BC patients with a limited number of $\mathrm{BM}$ who had undergone SRS for BM at the Institute of Oncology Ljubljana, and to identify clinical and pathohistological characteristics affecting their survival and local recurrence patterns.

\section{Materials and methods}

The study was approved by the institutional review board committee (approval number ERID-KSOPKR-0074/2020). We retrospectively reviewed the medical files of patients with metastatic BC who were consecutively treated with SRS for BM between April 2010 and December 2019. Brain disease was assessed by contrast-enhanced computed tomography (CT) and/or magnetic resonance imaging (MRI). All patients were presented at a multidisciplinary conference (MDT), consisting of a radiation oncologist, a medical oncologist, a radiologist, and an oncology surgeon. A neurosurgeon was consulted when required. The decision to treat $\mathrm{BM}$ with SRS was confirmed by the MDT. Patients underwent SRS with or without WBRT. Radiotherapy dose fractionation was left to the discretion of the treating radiation oncologist.

For most of the patients, a dedicated contrast-enhanced planning brain $C T$ was acquired, with a slice thickness of $1 \mathrm{~mm}$, using the BrainLAB (BrainLAB AG, Munich, Germany) thermoplastic mask and a frameless system for localization. The planning CT was co-registered with a post-gadolinium contrast-enhanced T1 weighted MRI. The use of contrast for the planning CT and the co-registered MRI improved the accuracy of image fusion. The gross tumor volume (GTV) was defined as the enhancing lesion on the post-gadolinium T1 weighted MRI. For postoperative cases, the GTV was defined as the resection bed plus any residual enhancement. The planning target volume (PTV) was defined as the GTV plus a $2 \mathrm{~mm}$ isotropic margin. For tumor $<3 \mathrm{~cm}$ a single fraction was delivered using RTOG 9005 protocol guidelines [14]. Single fractionated stereotactic radiosurgery was typically prescribed to a dose of 18-25 Gy. For larger tumors, particularly for tumors in eloquent areas, we used hypofractionated stereotactic radiosurgery in five fractions from $28-30$ Gy. The dose was normalized so the $100 \%$ isodose line encompassed nearly all (>95\%) of the PTV. All treatment plans were reviewed and approved by the treating radiation oncologist and physicist. SRS was performed using the True Beam TX Novalis Radiosurgery linear accelerator (Varian Medical Systems, inc.) with ExacTrac ${ }^{\circledast} \mathrm{X}$-ray $6 \mathrm{D}$ robotic image guidance. WBRT was performed as two-dimensional radiotherapy using a 6-MV photon beam by two laterally opposed standard fields or as a three-dimensional conformal radiotherapy technique.

Data were collected on general patient demographics, pathohistological and clinical characteristics of the primary BC tumor, systemic therapy (chemotherapy, endocrine treatment, or targeted therapy) used in both the adjuvant and metastatic 
settings. Local treatment details were reviewed including surgical notes and data regarding radiotherapy treatment. For the purpose of this study, patients' disease stage was classified using medical records, according to the seventh edition of the TNM Classification of Malignant Tumors [15]. The modified breast prognostic index (MB-GPA) was calculated for each patient [16]. Intrinsic BC subtypes were defined as Luminal A (ER+, HER2-, low Ki67, high PR+), Luminal B HER2- (ER+, HER2-, high Ki67 or low PR+), Luminal B HER2+ (ER+, HER2+, any PR, any Ki67), HER2+ (HER2+, PR-, ER-), and "Basal-like" (ER-, PR-, HER2-) [17]. "Basal-like" tumors were grouped with TNBC in our study. The study was approved by the local institutional review board committee.

\section{Statistical analysis}

Descriptive statistics were used to calculate typical measures in patients' demographic and clinical characteristics. Data were expressed as median with a range, and categorical data were expressed as counts and frequencies. A Pearson's chi-square test was used for comparing categorical variables between two groups. The primary endpoints were overall survival (OS) and local control. OS was calculated using the Kaplan-Meier method, measured from the time of BM diagnosis to the date of death or last follow-up. Progression-free survival (PFS) was defined as time to brain disease progression or development of new metastases (any brain recurrence) after the first local treatment. The log-rank test was used to compare OS between groups. All tests were two-sided, and a statistical level of significance was set to $p<0.05$. Univariate and multivariate Cox regressions were used to calculate the effect sizes [given as hazard ratios (HR), with 95\% confidence intervals (CI)]. Statistical analyses were carried out using IBM SPSS Statistics software version 26 (Statistical package for the Social Sciences Statistical Software; SPSS Inc, IBM Corporation, Armonk, New York).

\section{Results}

The cohort included 45 patients with $\mathrm{BC}$ who received treatment with SRS for 72 brain lesions. The median age at $\mathrm{BC}$ diagnosis, metastatic $\mathrm{BC}$ diagnosis, and $\mathrm{BM}$ diagnosis was 50.2 (29.6-83.0), 52.7 (29.6-88.0), and 55.9 (30.8-88.0) years, respectively. The median time from initial $\mathrm{BC}$ diagnosis to development of BM was 32.8 months (8.9224.4) and the median time from BM diagnosis to the first local treatment (surgery, SRS or WBRT) was 0.8 months (0-7.2). Patients for whom brain metastatic disease was the first sign of metastases were younger (53.3 years) and had a shorter median time from initial BC diagnosis to development of BM (19.3 months, range 8.9-178.0). In contrast, patients for whom extracranial disease spread was the first symptom of metastatic disease were older (56.7 years) and had a longer median time from initial BC diagnosis to development of BM (88.1 months, range 14.4-224.4), although the observed differences were not statistically significant. Patients' clinical, pathohistological, and treatment characteristics are presented in Table 1 . The median

Table 1. Patients' clinical and pathohistological characteristics

\begin{tabular}{|c|c|c|c|}
\hline \multicolumn{2}{|l|}{ Characteristic } & No. of patients & Percentage \\
\hline \multirow{4}{*}{ KPS at BM diagnosis } & 100 & 9 & $20.0 \%$ \\
\hline & $80-90$ & 28 & $62.2 \%$ \\
\hline & $60-70$ & 8 & $17.8 \%$ \\
\hline & $\leq 50$ & 0 & $0 \%$ \\
\hline \multirow{3}{*}{ Histology } & IDC & 40 & $88.9 \%$ \\
\hline & ILC & 4 & $8.9 \%$ \\
\hline & Other types & 1 & $2.2 \%$ \\
\hline \multirow{4}{*}{ Grade } & G1 & 0 & $0 \%$ \\
\hline & G2 & 15 & $33.3 \%$ \\
\hline & G3 & 29 & $64.4 \%$ \\
\hline & Missing values & 1 & $2.2 \%$ \\
\hline
\end{tabular}


Table 1. Patients' clinical and pathohistological characteristics

\begin{tabular}{|c|c|c|c|}
\hline \multicolumn{2}{|l|}{ Characteristic } & \multirow{2}{*}{$\begin{array}{c}\text { No. of patients } \\
24\end{array}$} & \multirow{2}{*}{$\begin{array}{c}\text { Percentage } \\
53.3 \%\end{array}$} \\
\hline \multirow{4}{*}{ Hormonal receptor } & ER-positive & & \\
\hline & ER-negative & 21 & $46.7 \%$ \\
\hline & PR-positive & 20 & $44.4 \%$ \\
\hline & PR-negative & 25 & $55.6 \%$ \\
\hline \multirow{3}{*}{ HER2 } & HER2-positive & 26 & $57.8 \%$ \\
\hline & HER2-negative & 17 & $37.8 \%$ \\
\hline & HER2-unknown & 2 & $4.4 \%$ \\
\hline \multirow{5}{*}{ Molecular subtype } & TNBC & 9 & $20.0 \%$ \\
\hline & Luminal A & 4 & $8.9 \%$ \\
\hline & Luminal B HER2- & 6 & $13.3 \%$ \\
\hline & Luminal B HER2+ & 14 & $31.1 \%$ \\
\hline & HER2+ & 12 & $26.7 \%$ \\
\hline \multirow{4}{*}{ Stage at $\mathrm{BC}$ diagnosis } & I & 4 & $8.9 \%$ \\
\hline & II & 13 & $28.9 \%$ \\
\hline & III & 25 & $55.6 \%$ \\
\hline & IV & 3 & $6.7 \%$ \\
\hline \multirow{4}{*}{ No. of BM } & 1 & 18 & $40.0 \%$ \\
\hline & 2 & 16 & $35.6 \%$ \\
\hline & 3 & 9 & $20.0 \%$ \\
\hline & $>3$ & 2 & $4.4 \%$ \\
\hline \multirow{5}{*}{ Extracranial disease } & Yes & 29 & $64.4 \%$ \\
\hline & No & 16 & $35.6 \%$ \\
\hline & Bone only & 3 & $6.7 \%$ \\
\hline & Visceral* and bone & 15 & $33.3 \%$ \\
\hline & Visceral* only & 11 & $24.4 \%$ \\
\hline \multirow{2}{*}{ Timing of metastatic spread } & Extracranial site first & 24 & $53.3 \%$ \\
\hline & Brain site first & 21 & $46.7 \%$ \\
\hline \multirow{4}{*}{ MB-GPA } & Class 1 & 3 & $6.7 \%$ \\
\hline & Class 2 & 12 & $26.6 \%$ \\
\hline & Class 3 & 23 & $51.1 \%$ \\
\hline & Class 4 & 7 & $15.6 \%$ \\
\hline \multirow{2}{*}{ Hormonal therapy } & Yes & 15 & $66.7 \%$ \\
\hline & No & 30 & $33.3 \%$ \\
\hline \multirow{2}{*}{ Chemotherapy } & Yes & 22 & $48.9 \%$ \\
\hline & No & 23 & $51.1 \%$ \\
\hline \multirow{2}{*}{ Anti-HER2 therapy } & Yes & 22 & $48.8 \%$ \\
\hline & No & 23 & $51.2 \%$ \\
\hline
\end{tabular}

No - number; BC — breast cancer; BM — brain metastases; KPS — Karnofsky performance status; ER - estrogen receptor; PR - progesterone receptor; HER2 - human epidermal growth receptor 2; IDC — invasive ductal carcinoma; ILC — invasive lobular carcinoma; WBRT — whole brain radiation therapy; MB-GPA - modified breast graded prognostic assessment; * *ung, liver, skin metastasis, other

number of systemic therapies received after BM diagnosis was 2 (range 0-6). The median number of treated $\mathrm{BM}$ was 2 (range 1-10). The total prescribed dose was 22 Gy (range 15-25) for single fraction- ated and 25 Gy (range 25-28) for hypofractionated SRS. All patients were treated with SRS during the course of the metastatic brain disease; however, local treatment sequence differed. The frequency of 
Table 2. Local treatment and median overall survival (OS) for patients treated with radiotherapy

\begin{tabular}{|c|c|c|c|c|}
\hline & & No. of patients (\%) & Median OS $(95 \% \mathrm{Cl})$, in months & p-value \\
\hline \multirow{2}{*}{ WBRT } & Yes & $34(75.5)$ & $26.2(10.7-41.7)$ & 0.360 \\
\hline & No & $11(24.4)$ & $36.3(13.6-58.9)$ & \\
\hline \multirow{3}{*}{ WBRT } & Before SRS & $16(35.6)$ & $30.4(5.5-55.3)$ & \multirow{3}{*}{0.113} \\
\hline & After SRS & $18(40.0)$ & $20.2(5.7-34.7)$ & \\
\hline & No WBRT & $11(24.4)$ & $36.3(13.6-58.9)$ & \\
\hline \multirow{2}{*}{ Surgery } & Yes & $17(37.8)$ & $36.1(25.9-46.3)$ & \multirow{2}{*}{0.023} \\
\hline & No & $28(62.2)$ & $15.9(11.8-20.0)$ & \\
\hline \multirow{2}{*}{$\begin{array}{l}\text { No. of local treatment } \\
\text { modalities }\end{array}$} & 2 & $23(51.1)$ & $15.9(7.7-24.1)$ & \multirow{2}{*}{0.130} \\
\hline & $\geq 2$ & $22(48.8)$ & $30.4(20.9-39.9)$ & \\
\hline \multirow{4}{*}{$\begin{array}{l}\text { Local treatment combi- } \\
\text { nations }\end{array}$} & SRS only & $5(11.1)$ & $36.3(7.6-64.9)$ & \multirow{4}{*}{0.094} \\
\hline & SRS + WBRT & $23(51.1)$ & $15.6(11.1-20.1)$ & \\
\hline & SRS + WBRT + Surgery & $11(24.4)$ & $36.1(23.3-48.9)$ & \\
\hline & SRS + Surgery & $6(13.3)$ & $33.6(0-69.2)$ & \\
\hline
\end{tabular}

No. - number, OS — overall survival; WBRT — whole brain radiation therapy; SRS — stereotactic radiosurgery

the first local treatment following BM detection was as follows: 24 (53.3\%) patients received SRS, 12 (26.7\%) received craniotomy (metastasectomy) and $9(20.0 \%)$ patients received SRS. Median time to SRS treatment since BM diagnosis was 1.6 months (range, 0.1-32.2). Other local treatment details are provided in Table 2.

\section{Survival}

The median follow-up time for the entire cohort was 20.2 months (range, 3.6-96.4). Median survivals from BM diagnosis and post-SRS were 27.6 months (95\% CI: 14.8-40.5) and 18.5 months (95\% CI: 11.1-25.8), respectively. The OS according to local treatment and $\mathrm{BC}$ intrinsic subtype is presented in Figure 1. Median one-year, two-year, and three-year survival rates were $55 \%, 41 \%$, and $16 \%$, respectively. At the time of study closeout date, 10 patients were still alive and 35 patients had died. Twenty-one (60\%) patients died from progressive brain disease, nine (25.7\%) from progressive extracranial disease, and one (2.8\%) patient from both intracranial and extracranial disease progression. The cause of death for three patients could not be determined. In a univariate analysis, improved survival was associated with the following pre-treatment or treatment-related factors: grade two primary $\mathrm{BC}$, hormonal-positive BC, HER2-positive $\mathrm{BC}$, controlled extracranial disease, metastasectomy, MP-GPA Class $\geq 3$, and receiving less than two lines of systemic therapy (Tab. 3). Upon multivariate Cox analysis only grade 2 primary BC tumors, surgical therapy, and utilization of more than one systemic therapy were significantly associated with improved median OS (Tab. 4). Patients that were treated in the first 10 years (until the end of 2014) were more likely to have had HER $2+$ BC (19 vs. $7 ; \mathrm{p}=0.001$ ) and less likely to be TNBC ( 1 vs. $8 ; \mathrm{p}=0.004$ ) than patients treated in the last five years of the observation period (2015-2019).

\section{Local control}

Imaging data were available for 43 patients at follow-up. Identification of any intracranial progression (local or distant) occurred in 34 (75.6\%) patients, nine patients did not have intracranial progression, and data for two patients were missing. The observed PFS for 43 patients was 29.3 months (range, 24.4-35.7) and varied according to HER2 status (HER2- vs. HER2+; 14.9 vs. 36.7 months, $\mathrm{p}<0.005$ ), ERstatus (ER-vs. ER+; $14.9 v$ s. 36.7 months; $\mathrm{p}<0.0005)$, and SRS biological dose $\left(\mathrm{BED}_{\alpha / \beta=10}\right)$ ( $<50$ Gy vs. $\geq 50$ Gy; 28.2 vs. 34.9 months; $\mathrm{p}=0.023$ ). There was a trend towards a longer PFS in patients who were treated initially with WBRT compared to patients who were treated with WBRT subsequently SRS or did not receive WBRT at all (38.7 vs. 27.1 months; $\mathrm{p}=0.054$ ). Median one-year, two-year, and three-year intracranial PFS rates were $85 \%, 63 \%$, and $33 \%$, respectively.

Any brain recurrence was seen in 25\%, 75\%, 83.3\%, 85.7\%, and $90.1 \%$ patients with Luminal A, 


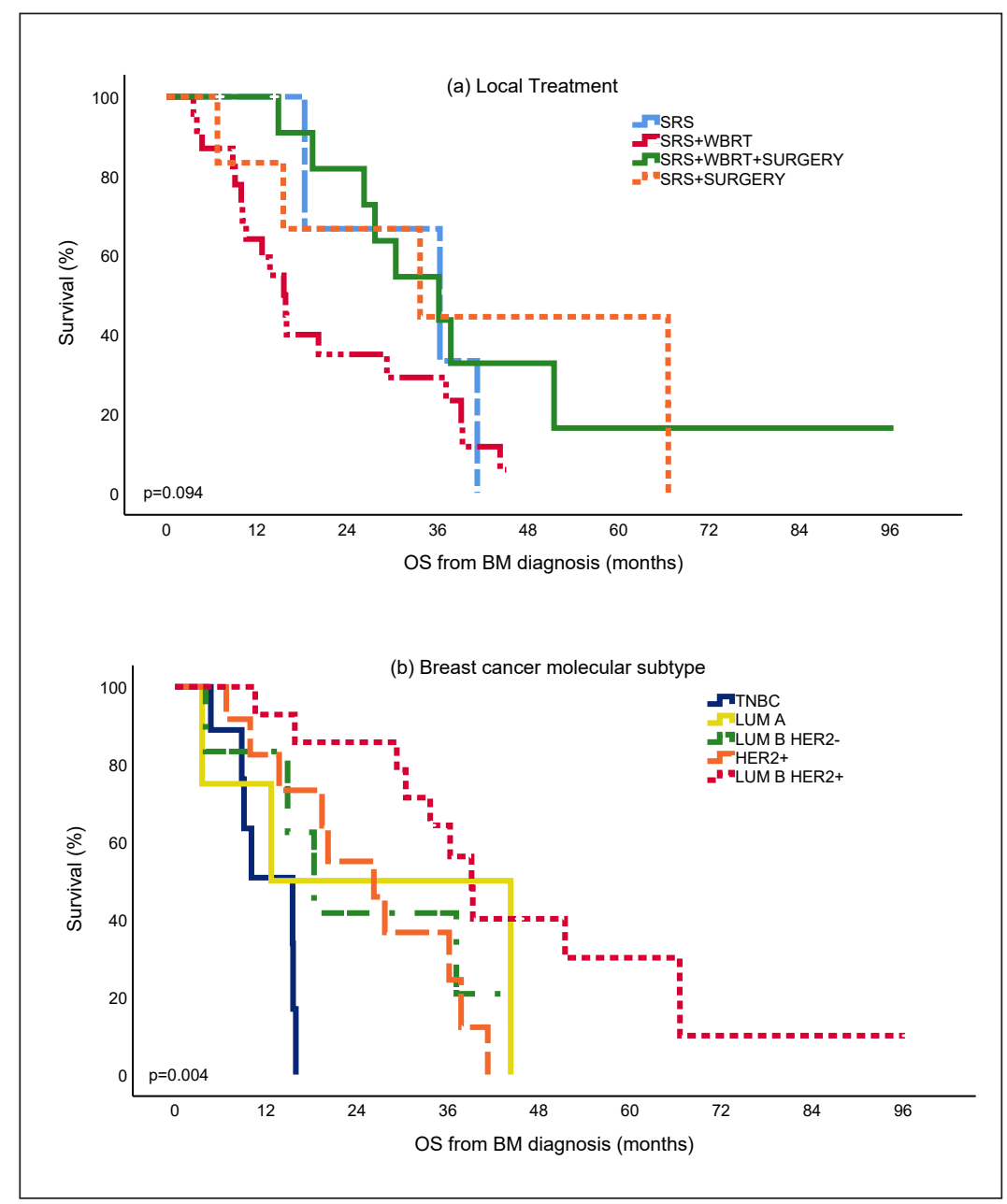

Figure 1. Overall survival from brain metastases diagnosis by (A) Local treatment and (B) Breast cancer molecular subtypes. OS - overall survival; BM — brain metastases; SRS — stereotactic radiosurgery, WBRT — whole brain radiation therapy; TNBC — triple negative breast cancer; LUM A — luminal A; LUM B — luminal B; HER2 — human epidermal growth receptor

Table 3. Prognostic factors influencing median overall survival (OS) times (univariate analysis)

\begin{tabular}{|c|c|c|c|c|}
\hline \multicolumn{2}{|l|}{ Variables } & $\begin{array}{c}\text { Median OS } \\
(95 \% \mathrm{Cl}) \text {, in months }\end{array}$ & HR $(95 \% \mathrm{CI})$ & p-value \\
\hline \multirow{2}{*}{ Age at BM diagnosis } & $<50$ & $27.6(12.2-43.1)$ & $1.1(0.5-2.3)$ & \multirow{2}{*}{0.793} \\
\hline & $\geq 50$ & $33.6(8.0-59.3)$ & Ref & \\
\hline \multirow{2}{*}{ Grade } & $\mathrm{G} 2$ & $39.3(35.7-42.8)$ & Ref & \multirow{2}{*}{0.004} \\
\hline & G3 & $15.9(9.7-22.1)$ & $3.1(1.4-7.1)$ & \\
\hline \multirow{2}{*}{ HER2 } & Positive & $36.1(27.5-44.7)$ & Ref & \multirow{2}{*}{0.046} \\
\hline & Negative & $15.5(14.2-16.8)$ & $2.1(1.0-4.4)$ & \\
\hline \multirow{2}{*}{ ER } & Positive & $37.1(29.6-44.6)$ & Ref & \multirow{2}{*}{0.002} \\
\hline & Negative & $15.6(8.2-22.9)$ & $3.1(1.5-6.6)$ & \\
\hline \multirow{5}{*}{ Intrinsic BC subtype } & Luminal B HER2+ & $39.1(34.1-44.1)$ & Ref & \multirow{5}{*}{0.004} \\
\hline & HER2+ & $26.2(14.2-38.3)$ & $3.1(1.2-8.1)$ & \\
\hline & Luminal A & $12.7(0-39.3)$ & $2.5(0.7-9.4)$ & \\
\hline & Luminal B HER2- & $18.3(11.1-25.6)$ & $2.2(0.7-7.4)$ & \\
\hline & TNBC & $15.5(7.8-23.2)$ & $11.0(3.3-37.2)$ & \\
\hline
\end{tabular}


Table 3. Prognostic factors influencing median overall survival (OS) times (univariate analysis)

\begin{tabular}{|c|c|c|c|c|}
\hline \multicolumn{2}{|l|}{ Variables } & $\begin{array}{c}\text { Median OS } \\
(95 \% \mathrm{Cl}) \text {, in months }\end{array}$ & HR $(95 \% \mathrm{Cl})$ & p-value \\
\hline \multirow{2}{*}{ KPS } & $\geq 80$ & $33.6(19.8-47.2)$ & Ref & \multirow{2}{*}{0.094} \\
\hline & $60-70$ & $9.1(0-21.6)$ & $2.2(0.9-5.1)$ & \\
\hline \multirow{2}{*}{$\begin{array}{l}\text { Systemic therapy } \\
\text { (number of lines) }\end{array}$} & $\leq 1$ & $13.7(7.1-20.3)$ & $2.2(1.0-4.6)$ & \multirow{2}{*}{0.042} \\
\hline & $\geq 2$ & $36.1(24.7-47.5)$ & Ref & \\
\hline \multirow{2}{*}{ Surgery } & Yes & $36.1(25.9-46.3)$ & Ref & \multirow{2}{*}{0.022} \\
\hline & No & $15.9(11.8-20.0)$ & $2.3(1.1-4.9)$ & \\
\hline \multirow{2}{*}{ Number of BM } & 1 or 2 & $29.3(14.7-43.7)$ & Ref & \multirow{2}{*}{0.657} \\
\hline & 3 & $26.2(12.2-40.2)$ & $1.2(0.5-2.7)$ & \\
\hline \multirow{2}{*}{$\mathrm{SRS} \mathrm{BED}_{\alpha / \beta=10}$} & $\geq 50$ Gy & $30.4(12.3-48.5)$ & Ref & \multirow{2}{*}{0.735} \\
\hline & $<50$ Gy & $27.6(9.5-45.8)$ & $1.2(0.5-2.6)$ & \\
\hline \multirow{2}{*}{$\begin{array}{l}\text { Extracranial disease } \\
\text { control }\end{array}$} & Yes & $87.9(16.1-159.9)$ & Ref & \multirow{2}{*}{0.033} \\
\hline & No & $25.2(14.0-36.3)$ & $2.2(1.1-4.5)$ & \\
\hline \multirow{4}{*}{ Extracranial disease } & No & $19.4(5.1-33.7)$ & Ref & \multirow{4}{*}{0.749} \\
\hline & Bone only & $36.3(/)$ & $1.0(0.2-4.7)$ & \\
\hline & Visceral* only & $27.6(5.2-50.1)$ & $1.5(0.6-3.7)$ & \\
\hline & Visceral* and bone & $29.2(3.0-55.4)$ & $0.9(0.4-2.2)$ & \\
\hline \multirow{2}{*}{ MB-GPA } & Class 1 or 2 & $14.9(10.3-19.4$ & $2.4(1.1-5.0)$ & \multirow{2}{*}{0.023} \\
\hline & Class 3 or 4 & $33.6(25.4-41.9)$ & Ref & \\
\hline \multirow{2}{*}{$\begin{array}{l}\text { Brain as the first } \\
\text { metastatic site }\end{array}$} & No & $36.1(25.3-47.0)$ & Ref & \multirow{2}{*}{0.533} \\
\hline & Yes & $15.6(9.1-22.1)$ & $1.2(0.6-2.4)$ & \\
\hline \multirow{2}{*}{ Year of BM diagnosis } & Before 2015 & $33.6(22.8-44.4)$ & Ref & \multirow{2}{*}{0.443} \\
\hline & 2015 and after & $15.9(12.2-19.6)$ & $1.3(0.6-2.7)$ & \\
\hline
\end{tabular}

G - grade; ER - estrogen receptor; HR — hazard ratio; OS — overall survival; BM — brain metastases; BC — breast cancer; TNBC — triple-negative breast cancer; KPS - Karnofsky Performance Status; HER2 — human epidermal growth receptor 2; MB-GPA — modified breast graded prognostic assessment; Gy — Gray; $\mathrm{BED}_{\alpha / \beta=10}$ - biological dose, SRS - stereotactic radiosurgery; *lung, liver, skin metastasis, other

Table 4. Prognostic factors influencing median overall survival (OS) times (multivariate Cox analysis)

\begin{tabular}{|l|c|c|}
\hline Variables & HR $(95 \% \mathrm{CI})$ & p-value \\
\hline MP-GPA Class $\geq 3$ & $0.3(0.1-1.3)$ & 0.392 \\
\hline Grade 2 & $0.1(0.1-0.6)$ & 0.005 \\
\hline HER+ BC & $0.5(0.2-1.7)$ & 0.966 \\
\hline ER+ BC & $0.8(0.2-3.4)$ & 0.529 \\
\hline Luminal B HER2+ & $0.7(0-22.1)$ & 0.661 \\
\hline $\begin{array}{l}\geq 2 \text { systemic therapy } \\
\text { lines received }\end{array}$ & $0.3(0.1-0.9)$ & 0.028 \\
\hline Surgery & $0.3(0.1-0.7)$ & 0.006 \\
\hline $\begin{array}{l}\text { Extracranial disease } \\
\text { controlled }\end{array}$ & $0.5(0.2-1.1)$ & 0.138 \\
\hline
\end{tabular}

HR - hazard ratio; OS - overall survival; BM — brain metastases; BC — breast cancer; KPS — Karnofsky Performance Status; HER2 — human epidermal growth receptor 2; ER - estrogen receptor; WBRT — whole brain radiation therapy

TNBC, Luminal B HER2-, Luminal B HER2+, and HER2+ molecular BC subtypes, respectively $(\mathrm{p}=0.074)$. Following SRS, local lesion progression was more prevalent in patients with HER2+ BC compared to HER2- BC $(72.0 \%$ vs. $31.3 \%$; $\mathrm{p}=0.022)$, and there was a tendency towards less common distal brain disease progression (36\% vs. $62.5 \% ; \mathrm{p}=0.097)$. Metastases secondary to Luminal B HER2+ BC, in comparison to all other BC molecular subtypes, showed an increased rate for any brain recurrence (HR: 3.3 95\% CI: 1.1-10.1; $\mathrm{p}=0.032$ ). However, in a multivariate analysis, after adjusting for SRS $B E D_{\alpha / \beta}=10$, systemic therapy received, ER status, and the use of WBRT or surgery, four factors remained significant for an increased risk of any intracranial recurrence, as follows: patients, younger than 50 years (HR: 4.2; 95\% CI: $1.3-36.3 ; \mathrm{p}=0.014$ ), grade 3 primary BC (HR: 3.7 ; 95\% CI: $1.1-13.2 ; \mathrm{p}=0.038)$, HER2+ receptor status (HR: 6.9; 95\% CI: 1.3-36.3; $\mathrm{p}=0.023$ ), and whether the brain was the first site of distant disease 
spread (HR: 4.7; 95\% CI: 1.6-14.5; $\mathrm{p}=0.006$ ). Local recurrence rates did not differ according to the year of treatment (before and after 2015; 76.2\% vs. $81.8 \%$; $\mathrm{p}=0.650)$.

Seventeen (45.9\%) patients developed radiographic leptomeningeal carcinomatosis (LMC) after SRS. The median time to LMC development after SRS was 11.5 months (range, 2.7-33.9). Out of 17 patients, whose follow-up imaging showed LMC, six had previous surgery and 11 were treated with radiation therapy only $(35.3 \%$ vs. $64.7 \% ; \mathrm{p}=$ $0.235)$. LMC was more often seen in HER2-negative $\mathrm{BC}(66.6 \%$ vs. $31.8 \%$; $\mathrm{p}=0.039)$. The longest time interval from SRS to LMC development was observed in patients who were initially treated with WBRT compared to patients who were treated with WBRT post-SRS or did not receive WBRT (22.8 vs. 8.3 months; $\mathrm{p}=0.036$ ).

\section{Discussion}

Metastases spreading from the primary $\mathrm{BC}$ tumor to the brain indicate a poor prognosis and represent a treatment challenge, despite recent systemic and local treatment innovations. In our study, we retrospectively explored our database and evaluated treatment outcomes for all patients with BC, who were consecutively treated with SRS for BM.

Median survival rates after BM diagnosis and post-SRS treatment (single fractionated or hypofractionated) were 27.6 and 18.5 months, respectively. Our results compare favorably with analogous studies, reporting OS after BM diagnosis in the range of 12-26 months [18-21] and post-SRS in the range of 15-19 months [22-24]. In a univariate analysis, improved survival was associated with grade 2 primary $\mathrm{BC}, \mathrm{HR}+$ and HER $2+\mathrm{BC}$, controlled extracranial disease, metastasectomy, MP-GPA Class $\geq 3$, and receiving more than two lines of systemic therapy. We have found out that patients with Luminal B HER2+ molecular subtype had the longest median OS. Due to bias in patient selection and a small study sample, our findings may not be truly representative. Nevertheless, our results demonstrated that patients with $\mathrm{BM}$ and HER2 + HR + primary BC have longer median OS compared to patients with HER- HR+ subtype and data are well in line with previous studies evaluating larger subsets of patients with BC BM [3-5]. One study also found that survival after BM was dependent on the HER2 status and not on the HR status; and was longer with HER2+ BC; suggesting the effect of novel anti-HER2 therapies [4]. In a multivariate Cox analysis, improved survival rates remained significant in moderately differentiated primary $\mathrm{BC}$, when surgery was part of the local treatment and when patients received at least two lines of systemic treatment. The importance of craniotomy was likewise demonstrated in a recent study, showing that longer survival rates might be achieved with surgery performed before receiving SRS [18]. In our study, WBRT, performed pre- or post-SRS did not influence OS rates, which is consistent with previous studies $[9,25]$. Other positive factors of improved OS following SRS, as described elsewhere in the literature, such as HER2+ $[18,23$, $24,26,27], \mathrm{HR}+$ disease [23, 24, 26], younger age of the patients [26] or extracranial disease control [23], were not associated with improved OS in our adjusted analysis, most probably due to small size of the study cohort and patient selection bias.

When analyzing local control outcomes, we observed worse intracranial control in patients with poorly differentiated and HER $2+$ primary BC tumors, in patients who were younger than 50 years of age and for whom BM occurred as the first symptom of metastatic disease, denoting aggressive tumor biology. Specifically, we found that HER2+ tumors more frequently recur locally and less often distally in the brain. Our observation is supported by findings in a similar study performed by Cagney et al. who found that HER2+ BC displayed poorer local control, whereas TNBC were more likely to recur distantly in the brain [6]. We did not find any differences in local or distant brain relapse according to the intrinsic BC subtype, which is most probably due to the low number of included cases. Grubb et al. found a considerable variation in the response to SRS on a per lesion basis when stratified by the BC subtype. Compared to luminal lesions (48\%), none of the HER2+ lesions exhibited a complete response at an average follow up time of six months, and only some of the TNBC lesions (35\%) responded. On the other hand, the authors did not find any differences in local lesion progression according to the BC subtype [27]. SRS dose escalation, boosting a tumor subvolume, the use of novel radiosensitizers and the development of new systemic therapies for $\mathrm{BM}$, have all been suggested to improve local control for patients with $\mathrm{BM}[6,28$, 
29]. However, more research is needed to prospectively investigate individualized SRS dose depending on BC molecular subtypes.

Interestingly, compared to HER2- tumors, we observed a statistically longer median time to brain disease progression after the first local treatment (absolute difference 19.8 months) in HER2+ disease. This observation can be explained by the effect of newer HER2-directed therapies, suggesting intracranial activity, which could prolong both the time to the development of BM and time to intracranial progression of $\mathrm{BM}$, following local treatment [30-32]. Parsai et al. showed that in patients with HER $2+$ BC subtypes, the use of lapatinib concurrently with SRS improved local control of BM, without increasing the rate of adverse events [32]. In our study, 22 out of 26 patients with the HER2+ BC subtype received anti-HER2 therapy in conjunction with SRS. The number of patients receiving lapatinib was too small to make any relevant analysis. A phase 2 study of WBRT/SRS with or without lapatinib in HER2+ $\mathrm{BC}$ subtype in patients with $\leq 10 \mathrm{BM}$ is ongoing to explore this topic further (ClinicalTrials.gov Identifier Number NCT01622868). Additional research, including both local radiation treatment strategies and systemic therapy, is warranted for all BC subtypes to identify therapies to optimize local control [33].

In our series, $45.9 \%$ of patients developed LMC post local treatment. The risk of LMC in patients with BM, treated with SRS was described previously, especially after BM resection [34]. WBRT or focal cranial radiotherapy might help to lower the LMC risk [35]. In our study, three-quarters of patients received WBRT, either upfront or following SRS. The addition of WBRT did not influence LMC, local lesion, or distant brain recurrence rates. Nevertheless, upfront WBRT prolonged time to develop any intracranial recurrence, but there was no difference in OS. The new tendency in the management of brain metastases is to avoid WBRT due to the toxicity and neurological deterioration [11]. However, this topic is still open to scientific debate.

We acknowledge downsides to our study, which might have influenced our results: retrospective data collection, small and unbalanced sample size, and not all medical charts were fully available at the time of study data collection. Furthermore, we did not systematically assess the neurological outcomes of our patients, which would improve the reporting of the treatment outcomes. However, our results, especially regarding improved OS rates, imply the cautious selection of BC patients throughout the observation period. In the future, multicentric prospective studies are warranted to further explore the use of SRS in patients with BC.

\section{Conclusions}

In this single-institutional retrospective study, evaluating patients with $\mathrm{BC}$, who underwent SRS for oligometastatic brain lesions, we observed improved survival rates. All patients were carefully evaluated and selected for SRS treatment by the multidisciplinary neurooncology team, which might partially contribute to the results. According to the intrinsic $\mathrm{BC}$ subtype, patients with Luminal B HER2+ BC had the longest estimated median OS, which exceeded three years. On the other hand, HER2+ local brain lesions were more likely to recur locally post-SRS and at the same time, the interval after first local brain treatment and brain lesion progression was the longest in patients with HER2+ tumors. SRS is a noninvasive and effective treatment modality and should be considered in all patients with $\mathrm{BC}$ who have $\mathrm{BM}$ and particularly in patients with HER 2+ BC subtypes because of their long-expected OS and duration of local treatment benefit. Our findings, supported by other investigators, warrant further explorations.

\section{Conflict of interest}

None declared.

\section{Financial disclosure}

None declared.

\section{References}

1. Berman AT, Thukral AD, Hwang WT, et al. Incidence and patterns of distant metastases for patients with earlystage breast cancer after breast conservation treatment. Clin Breast Cancer. 2013; 13(2): 88-94, doi: 10.1016/j. clbc.2012.11.001, indexed in Pubmed: 23218473.

2. Martin AM, Cagney DN, Catalano PJ, et al. Brain Metastases in Newly Diagnosed Breast Cancer: A Population-Based Study. JAMA Oncol. 2017; 3(8): 1069-1077, doi: 10.1001/ jamaoncol.2017.0001, indexed in Pubmed: 28301662.

3. Darlix A, Louvel G, Fraisse J, et al. Impact of breast cancer molecular subtypes on the incidence, kinetics and prognosis of central nervous system metastases in a large multicentre real-life cohort. Br J Cancer. 2019; 121(12): 
991-1000, doi: 10.1038/s41416-019-0619-y, indexed in Pubmed: 31719684.

4. Znidaric T, Gugic J, Marinko T, et al. Breast cancer patients with brain metastases or leptomeningeal disease: 10-year results of a national cohort with validation of prognostic indexes. Breast J. 2019; 25(6): 1117-1125, doi: 10.1111/ tbj.13433, indexed in Pubmed: 31286623.

5. Griguolo G, Jacot W, Kantelhardt E, et al. External validation of Modified Breast Graded Prognostic Assessment for breast cancer patients with brain metastases: A multicentric European experience. Breast. 2018; 37: 36-41, doi: 10.1016/j.breast.2017.10.006, indexed in Pubmed: 29073498.

6. Cagney DN, Lamba N, Montoya S, et al. Breast cancer subtype and intracranial recurrence patterns after braindirected radiation for brain metastases. Breast Cancer Res Treat. 2019; 176(1): 171-179, doi: 10.1007/s10549-01905236-6, indexed in Pubmed: 30982195.

7. Lee YT. Breast carcinoma: pattern of metastasis at autopsy. J Surg Oncol. 1983; 23(3): 175-180, doi: 10.1002/ jso.2930230311, indexed in Pubmed: 6345937.

8. Sundquist M, Brudin L, Tejler G. Improved survival in metastatic breast cancer 1985-2016. Breast. 2017; 31: 46-50, doi: 10.1016/j.breast.2016.10.005, indexed in Pubmed: 27810699.

9. Aoyama $\mathrm{H}$, Shirato $\mathrm{H}$, Tago $\mathrm{M}$, et al. Stereotactic radiosurgery plus whole-brain radiation therapy vs stereotactic radiosurgery alone for treatment of brain metastases: a randomized controlled trial. JAMA. 2006; 295(21): 2483-2491, doi: 10.1001/jama.295.21.2483, indexed in Pubmed: 16757720.

10. Soffietti R, Kocher M, Abacioglu UM, et al. Adjuvant wholebrain radiotherapy versus observation after radiosurgery or surgical resection of one to three cerebral metastases: results of the EORTC 22952-26001 study. J Clin Oncol. 2011; 29(2): 134-141, doi: 10.1200/JCO.2010.30.1655, indexed in Pubmed: 21041710.

11. Shinde A, Akhavan D, Sedrak M, et al. Shifting paradigms: whole brain radiation therapy versus stereotactic radiosurgery for brain metastases. CNS Oncol. 2019; 8(1): CNS27, doi: $10.2217 /$ cns-2018-0016, indexed in Pubmed: 30701987.

12. Grandhi R, Kondziolka D, Panczykowski D, et al. Stereotactic radiosurgery using the Leksell Gamma Knife Perfexion unit in the management of patients with 10 or more brain metastases. J Neurosurg. 2012; 117(2): 237-245, doi: 10.3171/2012.4.JNS11870, indexed in Pubmed: 22631694.

13. Brown PD, Gondi V, Pugh S, et al. for NRG Oncology. Hippocampal Avoidance During Whole-Brain Radiotherapy Plus Memantine for Patients With Brain Metastases: Phase III Trial NRG Oncology CC001. J Clin Oncol. 2020; 38(10): 1019-1029, doi: 10.1200/JCO.19.02767, indexed in Pubmed: 32058845.

14. Shaw $E$, Scott $C$, Souhami L, et al. Single dose radiosurgical treatment of recurrent previously irradiated primary brain tumors and brain metastases: final report of RTOG protocol 90-05. Int J Radiat Oncol Biol Phys. 2000; 47(2): 291-298, doi: 10.1016/s0360-3016(99)00507-6, indexed in Pubmed: 10802351.

15. Sobin LH, Gospodarowicz MK, Wittekind CH. TNM Classification of Malignant Tumours. 7th ed. International Union Against Cancer, John Wiley and Sons 2009.
16. Subbiah IM, Lei X, Weinberg JS, et al. Validation and Development of a Modified Breast Graded Prognostic Assessment As a Tool for Survival in Patients With Breast Cancer and Brain Metastases. J Clin Oncol. 2015; 33(20): 2239-2245, doi: 10.1200/JCO.2014.58.8517, indexed in Pubmed: 25987700.

17. Senkus E, Kyriakides S, Ohno S, et al. ESMO Guidelines Committee. Primary breast cancer: ESMO Clinical Practice Guidelines for diagnosis, treatment and follow-up. Ann Oncol. 2015; 26 Suppl 5: v8-30, doi: 10.1093/annonc/ mdv298, indexed in Pubmed: 26314782.

18. Abu-Khalaf M, Muralikrishnan S, Hatzis $C$, et al. Breast cancer patients with brain metastasis undergoing GKRS. Breast Cancer. 2019; 26(2): 147-153, doi: 10.1007/s12282018-0903-3, indexed in Pubmed: 30182250.

19. Cagney DN, Lamba N, Montoya S, et al. Breast cancer subtype and intracranial recurrence patterns after braindirected radiation for brain metastases. Breast Cancer Res Treat. 2019; 176(1): 171-179, doi: 10.1007/s10549-01905236-6, indexed in Pubmed: 30982195.

20. Cho E, Rubinstein L, Redman $M$, et al. Differentiation of overall survival by breast cancer tumor subtype following stereotactic radiosurgery for brain metastasis. J Clin Oncol. 2014;32(15_suppl): e11584-e11584, doi: 10.1200/ jco.2014.32.15_suppl.e11584.

21. Armstrong K, Ward J, Dunne M, et al. Linac-Based Radiosurgery for Patients With Brain Oligometastases From a Breast Primary, in the Trastuzumab Era-Impact of Tumor Phenotype and Prescribed SRS Dose. Front Oncol. 2019; 9: 377, doi: 10.3389/fonc.2019.00377, indexed in Pubmed: 31192116.

22. Wilson TG, Robinson T, MacFarlane $C$, et al. Treating Brain Metastases from Breast Cancer: Outcomes after Stereotactic Radiosurgery. Clin Oncol (R Coll Radiol). 2020; 32(6): 390-396, doi: 10.1016/j.clon.2020.02.007, indexed in Pubmed: 32131980.

23. Yang TJ, Oh JH, Folkert MR, et al. Outcomes and prognostic factors in women with 1 to 3 breast cancer brain metastases treated with definitive stereotactic radiosurgery. Int J Radiat Oncol Biol Phys. 2014; 90(3): 518-525, doi: 10.1016/j.ijrobp.2014.06.063, indexed in Pubmed: 25194669.

24. Cho E, Rubinstein L, Stevenson P, et al. The use of stereotactic radiosurgery for brain metastases from breast cancer: who benefits most? Breast Cancer Res Treat. 2015; 149(3): 743-749, doi: 10.1007/s10549-014-3242-x, indexed in Pubmed: 25638395.

25. Chang EL, Wefel JS, Hess KR, et al. Neurocognition in patients with brain metastases treated with radiosurgery or radiosurgery plus whole-brain irradiation: a randomised controlled trial. Lancet Oncol. 2009; 10(11): 1037-1044, doi: 10.1016/S1470-2045(09)70263-3, indexed in Pubmed: 19801201.

26. Kased N, Binder DK, McDermott MW, et al. Gamma Knife radiosurgery for brain metastases from primary breast cancer. Int J Radiat Oncol Biol Phys. 2009; 75(4): 1132-1140, doi: 10.1016/j.ijrobp.2008.12.031, indexed in Pubmed: 19345514.

27. Grubb CS, Jani A, Wu CC, et al. Breast cancer subtype as a predictor for outcomes and control in the setting of brain metastases treated with stereotactic radiosurgery. J Neurooncol. 2016; 127(1): 103-110, doi: 10.1007/s11060015-2014-8, indexed in Pubmed: 26615564. 
28. Abraham C, Garsa A, Badiyan SN, et al. Internal dose escalation is associated with increased local control for nonsmall cell lung cancer (NSCLC) brain metastases treated with stereotactic radiosurgery (SRS). Adv Radiat Oncol. 2018; 3(2): 146-153, doi: 10.1016/j.adro.2017.11.003, indexed in Pubmed: 29904739.

29. Tomé WA, Fowler JF. Selective boosting of tumor subvolumes. Int J Radiat Oncol Biol Phys. 2000; 48(2): 593-599, doi: 10.1016/s0360-3016(00)00666-0, indexed in Pubmed: 10974480.

30. Krop IE, Lin NU, Blackwell K, et al. Trastuzumab emtansine (T-DM1) versus lapatinib plus capecitabine in patients with HER2-positive metastatic breast cancer and central nervous system metastases: a retrospective, exploratory analysis in EMILIA. Ann Oncol. 2015; 26(1): 113-119, doi: 10.1093/annonc/mdu486, indexed in Pubmed: 25355722.

31. Stemmler HJ, Kahlert S, Siekiera W, et al. Characteristics of patients with brain metastases receiving trastuzumab for HER2 overexpressing metastatic breast cancer. Breast. 2006; 15(2): 219-225, doi:10.1016/j.breast.2005.04.017, indexed in Pubmed: 16026983.
32. Parsai S, Miller JA, Juloori A, et al. Stereotactic radiosurgery with concurrent lapatinib is associated with improved local control for HER2-positive breast cancer brain metastases. J Neurosurg. 2019; 132(2): 503-511, doi: 10.3171/2018.10.JNS182340, indexed in Pubmed: 30738402.

33. Shah N, Mohammad AS, Saralkar P, et al. Investigational chemotherapy and novel pharmacokinetic mechanisms for the treatment of breast cancer brain metastases. Pharmacol Res. 2018; 132: 47-68, doi: 10.1016/j.phrs.2018.03.021, indexed in Pubmed: 29604436

34. Ma R, Levy M, Gui B, et al. Risk of leptomeningeal carcinomatosis in patients with brain metastases treated with stereotactic radiosurgery. J Neurooncol. 2018; 136(2): 395-401, doi: 10.1007/s11060-017-2666-7, indexed in Pubmed: 29159778.

35. Prabhu RS, Turner BE, Asher AL, et al. A Multi-Institutional Analysis of Presentation and Outcomes for Leptomeningeal Disease Recurrence After Surgical Resection and Radiosurgery for Brain Metastases. Neuro Oncol. 2019 [Epub ahead of print], doi: 10.1093/neuonc/noz049, indexed in Pubmed: 30828727. 